\title{
Benzbromarone in the treatment of gout
}

\author{
Valderilio Feijó Azevedo ${ }^{1,2^{*}}$ (D) Igor Age Kos ${ }^{2}$, Ana Beatriz Vargas-Santos ${ }^{3}$, Geraldo da Rocha Castelar Pinheiro ${ }^{3}$ and \\ Eduardo dos Santos Paiva ${ }^{1}$
}

\begin{abstract}
Background: Benzbromarone is a uricosuric drug that has been used in the treatment of gout over the last 30 years. Due to its potent inhibition of the dominant apical (luminal) urate exchanger in the human proximal tubule URAT1, it reduces the urate reabsorption, diminishing serum urate levels and therefore preventing gout flares.

Main body of the abstract: Through several clinical trials, Benzbromarone has been proved effective and safe, inclusive in patients with chronic kidney disease and as combination therapy with allopurinol. Due to hepatotoxicity reports, it was withdrawn from the European market by the manufacturer, however many authors have questioned the product's withdrawal due to a lack of clinical evidence in order to support its hepatotoxicity. Benzbromarone is still available in several European countries, New Zealand, Brazil and several other countries. Despite the product's marketing over more than 20 years after the first hepatotoxicity reports, we have found only five reports in our literature search, and no prospective or retrospective study correlating hepatotoxicity with benzbromarone use.

Short conclusion: Benzbromarone is a safe and effective molecule for the treatment of gout. However, due to in vitro and in vivo data related to hepatotoxicity, it is prudent to prescribe it with some caution, especially for patients with an already known liver condition.
\end{abstract}

\section{Background}

Gout is the most prevalent inflammatory arthropathy in the general population [1]. It is triggered by an accumulation of monosodium urate crystals in the joint space, leading to inflammation and extreme pain [2]. The primary risk factor for its occurrence is hyperuricemia [2]. Therefore, this pathology reflects an intersection of metabolic and inflammatory disturbances $[3,4]$.

Patients experiencing gout flare complain of intense joint pain, but these events do not typically exceed one week [5]. However, in some cases, it is possible to observe a prolonged inflammatory response. This is often a result of inadequate treatment or refractory disease and can lead to the subsequent onset of tophi, macroscopic urate aggregates, among other substances, which can lead to joint destruction and physical limitation. This condition is known as tophaceous gout [2].

\footnotetext{
* Correspondence: valderilio@hotmail.com

'Universidade Federal do Paraná, Rua General Carneiro 181, Centro, Curitiba, Paraná, Brazil

Edumed Educação em Saúde, Rua Bispo Dom José, 2495, Curitiba, Paraná, Brazil

Full list of author information is available at the end of the article
}

Treatment for gout is divided in two steps: managing the gout flares and the long-term therapy [6]. The goal of the first step is to control pain and inflammation, primarily through anti-inflammatory agents [6]. In the second step, the therapeutic target is the reduction of the abnormally elevated levels of serum urate (sUA). Reducing sUA levels not only prevents recurrence but also addresses other systemic effects of hyperuricemia, such as elevated risk for cardiovascular incidents and chronic kidney disease [6-8].

Several strategies are used to reduce sUA. These include xanthine oxidase inhibitors such as allopurinol, uricases such as pegloticase, and uricosuric agents such as probenecid and benzbromarone [6].

Benzbromarone is a potent uricosuric agent that has been used to treat gout for over three decades [9]. Despite its demonstrated efficacy, this drug was never approved in the United States. It has been widely withdrawn from the market by its manufacturer, SanofiSynthelabo, who supplied the majority of the distributed product in Europe [10,11]. This withdrawal occurred after reports of hepatotoxicity. However, some authors maintain that there is not sufficient evidence to justify

(c) The Author(s). 2019 Open Access This article is distributed under the terms of the Creative Commons Attribution 4.0 International License (http://creativecommons.org/licenses/by/4.0/), which permits unrestricted use, distribution, and 
the drug's withdrawal $[10,11]$. Benzbromarone is still approved and marketed in several countries, including Brazil, New Zealand and some European countries [12]. In the last few years, Brazil has experienced a gap in the product's manufacturing that reduced benzbromarone availability in the country. This restricted availability has led to considerable drops in the number of benzbromarone prescriptions given in Brazil and consequently diminished prescribers' experience with the drug.

Therefore, this article aims to review the pharmacological properties of benzbromarone as well to review the evidence of its efficacy and safety.

\section{Pharmacological Properties}

Benzbromarone is a benzofuran derivative that shares structural similarities with amiodarone and benzarona [13]. It is an uricosuric drug that functions by increasing urate excretion in the kidney's proximal tubule through inhibition of the dominant apical (luminal) urate exchanger in the human proximal tubule URAT1 [14, 15]. This blockage reduces urate reabsorption, increasing its elimination via the urine. In vitro studies have demonstrated that benzbromarone reduces urate reabsorption by $93 \%$ [14]. The ion transporters OAT 1 and OAT 3 are responsible for urate secretion in the kidney tubules. Benzbromarone appears to have a mild effect on OAT 1, but not on OAT 3 [16].

Benzbromarone has a hepatic metabolism, mainly through cytochrome P450 2C9 (CYP2C9). Its primary metabolites are 6-hydroxybenzbromarone, which is related to its uricosuric effect, and 1'-hydroxybenzbromarone, whose uricosuric effect is unknown [17]. The intact drug's half-life is approximately $3 \mathrm{~h}$; however, its active metabolite can persist for $30 \mathrm{~h}$, resulting in a prolonged effect [18]. Both metabolites are excreted through the bile and feces [18].

\section{Efficacy evidence}

Several studies and meta-analyses have demonstrated benzbromarone's efficacy in reducing sUA levels and preventing gout flares. The usual dosage varies between $50 \mathrm{mg}$ and $200 \mathrm{mg}$ daily [16, 19-21].

In healthy subjects and in subjects with hyperuricemia, the drug was shown to increases urate clearance by $500 \%$, leading to an average reduction in sUA of $25-50 \%$ $[16,22,23]$. A study from 2002 compared the efficacy of $100 \mathrm{mg}$ benzbromarone to $300 \mathrm{mg}$ allopurinol. The study reported a significantly larger reduction in sUA from the benzbromarone $(9.53+/-1.48$ to $4.05+/-0.87 \mathrm{mg} / \mathrm{dl})$ compared to allopurinol $(9.89+/-1.43 \mathrm{mg} / \mathrm{dl}$ to 5.52 $+/-0.83 \mathrm{mg} / \mathrm{dl}$ ) [24]. Another study, published in 1999, demonstrated a higher efficacy of benzbromarone over allopurinol in patients taking diuretics and in patients with high baseline sUA levels [25]. Finally, a third study from 2009 reported that there was no difference in the success rates for gout treatment between the two drugs after dose scaling [26]. In summary, evidence suggests that both treatments have similar efficacies in reducing gout flares and tophi reduction, once the optimal sUA is achieved. The primary difference between the two is their rate of sUA reduction [27].

Benzbromarone has also been shown to be effective in patients who have failed to achieve sUA goals with allopurinol treatment. One randomized controlled trial published in 2009 demonstrated the and higher efficacy and tolerance profile of $200 \mathrm{mg}$ of benzbromarone compared to $2 \mathrm{~g}$ of probenecid [28]. Other studies have also reported the efficacy of combined allopurinol and benzbromarone treatments as well as the efficacy of giving benzbromarone to patients demonstrating poor control with allopurinol monotherapy $[29,30]$.

One thirteen-year cohort compared the effectiveness of allopurinol, febuxostat and benzbromarone on kidney function and disease progression to explore hyperuricemia treatments for patients with chronic kidney disease [31]. The median values of the mean daily for dosage Febuxostat, Benzbromarone and Allopurinol were $40.0 \mathrm{mg} /$ day 88.9 $\mathrm{mg} /$ day $100.0 \mathrm{mg} /$ day respectively, and the median estimated glomerular filtration rate (eGRF) was 22.3 (10.734.7) 37.7 (24.8-49.1) and 20.3 (11.4-33.8) respectively. The study reported that benzbromarone was more effective in reducing progression to dialysis, with a hazard ratio of 0.5. Furthermore, the patients in the benzbromarone and febuxostate groups had reduced progression to end-stage kidney disease compared with the allopurinol group [31].

\section{Safety evidence}

Benzbromarone was withdrawn from the market by Sanofi-Synthelabo in 2003, after reports of serious hepatotoxicity [10]. The first of these reports was from the Netherlands and was published in 1994, followed by three Japanese reports in the early 2000s [32-35]. In our literature review, we found no published reports on this topic in the Latin American databases Lilacs and SciELO. Our search found only one additional report, which was published in 2016 in Japan [36]. A total of 11 cases of hepatotoxicity related to benzbromarone were reported by the Periodic Safety Update Report (PSUR) between 1995 and 2000. Of the reported total of 11 patients, 9 died [16] The four published cases are assumed to be the total number. Several laboratory essays have tried to clarify the mechanism for the reported hepatotoxicity. Furthermore, many cohort, meta-analysis and systematic reviews have evaluated additional safety aspects of benzbromarone.

\section{Studies evaluating Benzbromarone's safety}

A carefully performed review published in 2008 examined the risk-benefit of benzbromarone use and the frequency of drug-related hepatotoxicity. The review 
reported that hepatotoxicity occurred in 1:17000 patients [16]. The review found no other publications reporting severe adverse events beyond those already cited. In our literature search, we found several other studies published in or after 2008 that directly or indirectly evaluated the safety of benzbromarone [19-21, 26, 28, 37-39]. Among these, a one-year prospective analysis of 123 patients in New Zealand and a three-year Chinese retrospective study both reported no serious events related to benzbromarone treatment $[19,21]$. Two other systematic reviews reported similar results [20,38].

Regarding benzbromarone use in patients with chronic kidney disease, there is satisfactory evidence for its efficacy with creatinine clearance levels of at least $20 \mathrm{ml} /$ $\mathrm{min} / 1.73 \mathrm{~m} 2[19,25]$. Furthermore, studies have shown an incidence of approximately $3 \%$ for urolithiasis in patients treated with benzbromarone $75-120 \mathrm{mg} / \mathrm{d}$ or a median dose of $100 \mathrm{mg} /$ day $[15,19]$. The 5 published reported cases include 2 deaths related to fulminant hepatitis, one case of fulminant hepatitis with response to steroid treatment, one case of hepatotoxicity responsive to withdrawal and one case of auto-immune hepatitis in which benzbromarone was co-administered with camostat mesilate [32-36].

\section{A mechanism for hepatotoxicity: animal models and in vitro studies}

Mitochondrial toxicity Several studies have tried to explain the potential mechanisms involved in benzbromarone's hepatotoxicity. Some studies have hypothesized that it is due to the structural similarities between this drug and amiodarone, whose hepatotoxicity is better defined [13, 40, 41]. Studies in mouse hepatocytes and isolated mitochondria have reported that these molecular structures may inhibit the mitochondrial respiratory chain and beta-oxidation, produce oxidative phosphorylation uncoupling, and generate reactive oxygen species, leading to mitochondrial edema, apoptosis and cellular necrosis [13]. A study in HepG2 cells and primary human hepatocytes demonstrated similar mitochondrial toxicity for Benzbromarone [41].

Metabolization products In regard to hepatic metabolization, one study evaluated the toxicity of the metabolites 6-hydroxybenzbromarone and 1'-hydroxybenzbromarone in the human hepatocarcinoma cell line FLC-4. There was a correlation between cytotoxicity and benzbromarone's metabolic activation of CYP3A4, producing 1'hydroxybenzbromarone [42]. The metabolite 6hydroxybenzbromarone was not correlated to toxicity in these cells. Another possible mechanism could be related to glutathione adduct formation through sequential metabolization. One study has reported that the metabolization of 6-hydroxybenzbromarone through CYP2C9 in human microsomes generates the intermediate catechol 5,6-dihydroxybenzobromarone. The final metabolite of this molecule is ortho-quinone, which may be related to hepatotoxicity [43]. Other studies have reported the presence of an intermediate epoxide metabolite, which has been demonstrated to be hepatotoxic in murine models $[44,45]$. Another study reported that the addition of a fluorine molecule in the 6 position of benzbromarone reduced the production of these metabolites, reducing its toxicity in mice [46].

Another metabolite, 1',6-dihydroxybenzbromarone, may also be related to toxicity through the formation of reactive intermediates that bind to glutathione. These intermediates were correlated with hepatic injury in mice [47]. 2,6-dibromohydroquinone (DBH) and mono-debrominated catechol were also identified as possible intermediates of benzbromarone and are highly toxic in HepG2 cells [48].

Other mechanisms Some toxicological studies have demonstrated peroxisome proliferation (a mechanism that could be related to carcinogenesis) in the livers of mice that received benzbromarone; however, this result has not been verified in human hepatocytes [49-51].

Additionally, a recent study has demonstrated that benzbromarone worsened hepatic steatosis in obese mice while having little effect in normal-weight mice [52]. The same study also reported an increase in intracellular lipid accumulation in HepG2 cells through the regulation of genes involved in lipid metabolism. This accumulation led to increased apoptosis in these cells [52].

\section{Discussion}

The efficacy of benzbromarone in treating gout has been well established. It was verified by several trials, including in patients who did not respond to allopurinol treatment. Despite its safety being demonstrated in clinical trials and meta-analyses, hepatotoxicity reports raised concerns about benzbromarone and led to its withdrawal from the market. Clinical trials have a low sensitivity for detecting rare adverse events, and thus post marketing surveillance-pharmacovigilance-is necessary [53]. Some authors have questioned the lack of convincing evidence for this product's withdrawal, attributing it to a fear of lawsuits against the pharmaceutical company [10]. Indeed, there is scarce clinical evidence for hepatotoxicity from benzbromarone treatment.

Despite the continued marketing of the drug in several countries, our literature search found only one additional report of benzbromarone toxicity (beyond those that preceded the product's withdrawal). In this report, while possible, benzbromarone toxicity remains 
questionable, especially in light of the probable causal role of the co-administered camostat mesilate, which had demonstrated a positive lymphocyte stimulation test, in contrast to benzbromarone. However, it was not possible to entirely rule out benzbromarone [36].

Despite the toxicity reports, in 2006, the European League Against Rheumatism (EULAR) published recommendations for the treatment of gout that did not prohibit prescribing benzbromarone [54]. In fact, they recommended its use in patients with mild to moderate chronic kidney disease.

In vitro evidence and experience with animal models have provided several possible mechanisms related to benzbromarone hepatotoxicity. Together with the hepatotoxicity reports, these studies suggest that some caution should be taken when prescribing benzbromarone. However, considering its high efficacy and affordable price, its use is still encouraged [54].

Some authors have proposed empirical measures that could help prevent hepatotoxicity, including avoiding its use in patients with known hepatic disease, initiating treatment with low dose regimens, monitoring liver enzymes during treatment, and avoiding the association of benzbromarone with other hepatotoxic medicines, herbal medicines and alcohol ingestion [16]. Notably, however, among the reported hepatotoxicity cases, none of the patients had a clear history of hepatic disease, though one had a history of jaundice with no apparent cause. Therefore, it seems prudent to avoid its prescription in patients with hepatic disease, despite the limited evidence of safe administration in cirrhotic patients [55]. Considering the recent studies demonstrating a worsening of steatosis in obese mice, it also seems reasonable to prescribe it cautiously in patients who already have hepatic steatosis [52]. Four of the reported cases were treated for asymptomatic hyperuricemia, which normally does not provide a clear indication of treatment [32-34, 36, 52]. Hence, another indication proposed by some authors would be avoiding benzbromarone in patients with this condition [16].

\section{Conclusions}

Benzbromarone is an effective drug for lowering sUA levels and preventing the gout flares. Additionally, it has also been shown to be successful in combination with allopurinol treatment in patients who do not respond properly to monotherapy. In general, its adverse events are mild, and it can be prescribed to patients with chronic kidney disease. Due to previous hepatotoxicity reports and experimental evidence gathered so far, it is reasonable to recommend active monitoring of hepatic function and enzymes and avoid its use in patients with known hepatic disease or asymptomatic hyperuricemia.
Authors' contributions

All authors have contributed equally to the development of the manuscript. All authors read and approved the final manuscript.

\section{Funding}

Not applicable

Availability of data and materials

Not applicable

Ethics approval and consent to participate

Not applicable

\section{Consent for publication}

Not applicable

\section{Competing interests}

Valderilio Azevedo: has received grant and speaker fees from AbbVie and Pfizer, speaker fees from UCB, Janssen, BMS, and advisory board fees from Pfizer.

Geraldo da Rocha Castelar Pinheiro: Has received consulting fees from Grünenthal.

Ana Beatriz Vargas-Santos has received speaking fee (USD $<1,000.00)$ and supporting for international medical events from Grünenthal.

The other authors declare to have no competing interests.

\section{Author details}

'Universidade Federal do Paraná, Rua General Carneiro 181, Centro, Curitiba, Paraná, Brazil. ${ }^{2}$ Edumed Educação em Saúde, Rua Bispo Dom José, 2495, Curitiba, Paraná, Brazil. ${ }^{3}$ Universidade Estadual do Rio de Janeiro, Rio de Janeiro, Brazil.

Received: 27 September 2018 Accepted: 19 July 2019

Published online: 07 August 2019

\section{References}

1. Roddy E, Doherty M. Epidemiology of gout. Arthritis Res Ther BioMed Central. 2010;12(6):223

2. Perez-Ruiz F, Dalbeth N, Bardin T. A review of uric acid, crystal deposition disease, and gout. Adv Ther Springer. 2015;32(1):31-41.

3. Bardin T, Richette P. Impact of comorbidities on gout and hyperuricaemia: an update on prevalence and treatment options. BMC Med BioMed Central. 2017;15(1):123.

4. Kuo C-F, Grainge MJ, Mallen C, Zhang W, Doherty M. Comorbidities in patients with gout prior to and following diagnosis: case-control study. Ann Rheum Dis. 2016;75(1):210-7 BMJ Publishing Group.

5. Cronstein BN, Sunkureddi P. Mechanistic aspects of inflammation and clinical management of inflammation in acute gouty arthritis. J Clin Rheumatol. 2013;19(1):19-29 NIH Public Access.

6. Azevedo VF, Lopes MP, Catholino NM, Dos E, Paiva S, Araújo A, et al. Revisão crítica do tratamento medicamentoso da gota no Brasil critical revision of the medical treatment of gout in Brazil. Rev Bras Reumatol. 2017;57:346-55.

7. Li L, Yang C, Zhao Y, Zeng X, Liu F, Fu P. Is hyperuricemia an independent risk factor for new-onset chronic kidney disease?: A systematic review and meta-analysis based on observational cohort studies. BMC Nephrol. 2014;15: 122 BioMed Central.

8. Filippatos GS, Ahmed MI, Gladden JD, Mujib M, Aban IB, Love TE, et al. Hyperuricaemia, chronic kidney disease, and outcomes in heart failure: potential mechanistic insights from epidemiological data. Eur Heart J. 2011; 32(6):712-20 Oxford University Press.

9. Perez-Ruiz F, Alonso-Ruiz A, Calabozo M, Herrero-Beites A, García-Erauskin G, Ruiz-Lucea E. Efficacy of allopurinol and benzbromarone for the control of hyperuricaemia. A pathogenic approach to the treatment of primary chronic gout. Ann Rheum Dis. 1998;57(9):545-9 BMJ Publishing Group.

10. Jansen TLTA, Reinders MK, van Roon EN, Brouwers JRBJ. Benzbromarone withdrawn from the European market: another case of \&quot;absence of evidence is evidence of absence\&quot;? Clin Exp Rheumatol. 22(5):651.

11. Benzbromarone [Internet]. [cited 2018 May 24]. Available from: https:// livertox.n/m.nih.gov/Benzbromarone.htm.

12. Benzbromarone - Drugs.com [Internet]. [cited 2018 May 29]. Available from: https://www.drugs.com/international/benzbromarone.html 
13. Kaufmann P, Török M, Hänni A, Roberts P, Gasser R, Krähenbühl S. Mechanisms of benzarone and benzbromarone-induced hepatic toxicity. Hepatology. 2005;41(4):925-35 Wiley-Blackwell.

14. Enomoto A, Kimura $H$, Chairoungdua A, Shigeta $Y$, Jutabha $P$, Ho Cha S, et al. Molecular identification of a renal urate-anion exchanger that regulates blood urate levels. Nature. 2002;417(6887):447-52.

15. Masbernard A, Giudicelli CP. Ten years' experience with benzbromarone in the management of gout and hyperuricaemia. S Afr Med J. 1981;59(20): 701-6.

16. Lee M-HH, Graham GG, Williams KM, Day RO. A benefit-risk assessment of benzbromarone in the treatment of gout. Was its withdrawal from the market in the best interest of patients? Drug Saf. 2008;31(8):643-65.

17. Vries JX, Walter-Sack I, Ittensohn A, Weber E, Empl H, Gresser U, et al. Benzbromarone hydroxylation in man: defective formation of the 6hydroxybenzbromarone metabolite. Clin Investig. 1993;71(11):947-52 Springer-Verlag.

18. Jain AK, Ryan JR, McMahon FG, Noveck RJ. Effect of single oral doses of benzbromarone on serum and urinary uric acid. Arthritis Rheum. 17(2):149-57.

19. Stamp LK, Haslett J, Frampton C, White D, Gardner D, Stebbings S, et al. The safety and efficacy of benzbromarone in gout in Aotearoa New Zealand. Intern Med J. 2016:46(9):1075-80.

20. Li S, Yang H, Guo Y, Wei F, Yang X, Li D, et al. Comparative efficacy and safety of urate-lowering therapy for the treatment of hyperuricemia: a systematic review and network meta-analysis. Sci Rep. 2016;6(1):33082.

21. Zhou Q, Su J, Zhou T, Tian J, Chen J, Zhu J. A study comparing the safety and efficacy of febuxostat, allopurinol, and benzbromarone in Chinese gout patients: a retrospective cohort study. Int J Clin Pharmacol Ther. 2017:55(02):163-8.

22. Moriwaki Y, Yamamoto T, Takahashi S, Hada T, Higashino K. Analysis of uric acid transport in renal tubules using benzbromarone and pyrazinamide. Int J Clin Pharmacol Ther Toxicol. 1990;28(2):84-8.

23. Bluestone $\mathrm{R}$, Klinenberg J, Lee IK. Benzbromarone as a long-term uricosuric agent. Adv Exp Med Biol. 1980;122A:283-6.

24. Hanvivadhanakul P, Akkasilpa S, Deesomchok U. Efficacy of benzbromarone compared to allopurinol in lowering serum uric acid level in hyperuricemic patients. J Med Assoc Thail. 2002;85(Suppl 1):S40-7.

25. Perez-Ruiz F, Calabozo M, Fernandez-Lopez MJ, Herrero-Beites A, Ruiz-Lucea E, Garcia-Erauskin G, et al. Treatment of chronic gout in patients with renal function impairment: an open, randomized, actively controlled study. J Clin Rheumatol. 1999:5(2):49-55.

26. Reinders MK, Haagsma C, Jansen TLTA, van Roon EN, Delsing J, van de Laar MAFJ, et al. A randomised controlled trial on the efficacy and tolerability with dose escalation of allopurinol 300-600 mg/day versus benzbromarone 100-200 mg/day in patients with gout. Ann Rheum Dis. 2009;68(6):892-7.

27. Perez-Ruiz F, Calabozo M, Pijoan II, Herrero-Beites AM, Ruibal A. Effect of urate-lowering therapy on the velocity of size reduction of tophi in chronic gout. Arthritis Rheum. 2002;47(4):356-60.

28. Reinders MK, van Roon EN, Jansen TLTA, Delsing J, Griep EN, Hoekstra M, et al. Efficacy and tolerability of urate-lowering drugs in gout: a randomised controlled trial of benzbromarone versus probenecid after failure of allopurinol. Ann Rheum Dis. 2008:68(1):51-6.

29. Kumar S, Ng J, Gow P. Benzbromarone therapy in management of refractory gout. N Z Med J. 2005;118(1217):U1528.

30. Azevedo VF, Buiar PG, Giovanella LH, Severo CR, Carvalho M. Allopurinol, benzbromarone, or a combination in treating patients with gout: analysis of a series of outpatients. Int J Rheumatol. 2014;2014:263720.

31. Chou H-W, Chiu H-T, Tsai C-W, Ting I-W, Yeh H-C, Huang H-C, et al. Comparative effectiveness of allopurinol, febuxostat and benzbromarone on renal function in chronic kidney disease patients with hyperuricemia: a 13year inception cohort study. Nephrol Dial Transplant. 2017;33(9):1620-7.

32. Suzuki T, Suzuki T, Kimura M, Shinoda M, Fujita T, Miyake N, et al. A case of fulminant hepatitis, possibly caused by benzbromarone. Nihon Shokakibyo Gakkai Zasshi. 2001;98(4):421-5

33. Wagayama H, Shiraki K, Sugimoto K, Fujikawa K, Shimizu A, Takase K, et al, Fatal fulminant hepatic failure associated with benzbromarone. J Hepatol. 2000;32(5):874

34. van der Klauw MM, Houtman PM, Stricker BHC, Spoelstra P. Hepatic injury caused by benzbromarone. J Hepatol. 1994;20(3):376-9.

35. Arai M, Yokosuka O, Fujiwara K, Kojima H, Kanda T, Hirasawa H, et al. Fulminant hepatic failure associated with benzbromarone treatment: a case report. J Gastroenterol Hepatol. 2002;17(5):625-6.
36. Kumagai J, Kanda T, Yasui S, Haga Y, Sasaki R, Nakamura M, et al. Autoimmune hepatitis following drug-induced liver injury in an elderly patient. Clin J Gastroenterol. 2016;9(3):156-9.

37. Yu H, Liu X, Song Y, Cheng J, Bao H, Qin L, et al. Safety and efficacy of Benzbromarone and Febuxostat in hyperuricemia patients with chronic kidney disease: a prospective pilot study. Clin Exp Nephrol. 2018;14.

38. Castrejon I, Toledano E, Rosario MP, Loza E, Pérez-Ruiz F, Carmona L. Safety of allopurinol compared with other urate-lowering drugs in patients with gout: a systematic review and meta-analysis. Rheumatol Int. 2015;35(7):1127-37.

39. Kydd AS, Seth R, Buchbinder R, Edwards CJ, Bombardier C. Uricosuric medications for chronic gout. Cochrane Database Syst Rev. 2014;11:CD010457.

40. Lewis JH, Ranard RC, Caruso A, Jackson LK, Mullick F, Ishak KG, et al. Amiodarone hepatotoxicity: prevalence and clinicopathologic correlations among 104 patients. Hepatology. 1989;9(5):679-85.

41. Felser A, Lindinger PW, Schnell D, Kratschmar DV, Odermatt A, Mies S, et al. Hepatocellular toxicity of benzbromarone: effects on mitochondrial function and structure. Toxicology. 2014;324:136-46.

42. Kobayashi K, Kajiwara E, Ishikawa M, Mimura H, Oka H, Ejiri Y, et al. Cytotoxic effects of Benzbromarone and its 1'-Hydroxy metabolite in human Hepatocarcinoma FLC4 cells cultured on micro-space cell culture plates. Drug Metab Pharmacokinet. 2013;28(3):265-8.

43. McDonald MG, Rettie AE. Sequential metabolism and bioactivation of the Hepatotoxin Benzbromarone: formation of glutathione adducts from a catechol intermediate. Chem Res Toxicol. 2007;20(12):1833-42.

44. Wang K, Wang H, Peng Y, Zheng J. Identification of Epoxide-Derived Metabolite(s) of Benzbromarone. Drug Metab Dispos. Am Soc Pharmacol Exp Ther. 2016;44(4):607-15.

45. Wang H, Feng Y, Wang Q, Guo X, Huang W, Peng Y, et al. Cysteine-based protein adduction by epoxide-derived metabolite(s) of Benzbromarone. Chem Res Toxicol American Chemical Soc. 2016;29(12):2145-52.

46. Wang H, Peng Y, Zhang T, Lan Q, Zhao H, Wang W, et al. Metabolic epoxidation is a critical step for the development of Benzbromaroneinduced hepatotoxicity. Drug Metab Dispos. 2017:45(12):1354-63.

47. Yoshida M, Cho N, Akita H, Kobayashi K. Association of a reactive intermediate derived from 1',6-dihydroxy metabolite with benzbromaroneinduced hepatotoxicity. J Biochem Mol Toxicol. 2017;31(10):e21946.

48. Kitagawara Y, Ohe T, Tachibana K, Takahashi K, Nakamura S, Mashino T. Novel bioactivation pathway of Benzbromarone mediated by cytochrome P450. Drug Metab Dispos. 2015;43(9):1303-6.

49. Bichet N, Cahard D, Fabre G, Remandet B, Gouy D, Cano J-P. Toxicological studies on a benzofuran derivative: III. Comparison of peroxisome proliferation in rat and human hepatocytes in primary culture. Toxicol Appl Pharmacol. 1990;106(3):509-17 Academic Press.

50. Parzefall W, Schuppler J, Barthel G, Meyer-Rogge B, Schulte-Hermann R. Toxicological studies on a benzofurane derivative: I. A comparative study with phenobarbital on rat liver. Toxicol Appl Pharmacol. 1990;106(3):482-99 Academic Press.

51. Butler EG, Ichida T, Maruyama H, Schulte-Hermann R, Williams GM. Toxicological studies on a benzofurane derivative: II. Demonstration of peroxisome proliferation in rat liver. Toxicol Appl Pharmacol. 1990;106(3): 500-8 Academic Press.

52. Sun P, Zhu J-J, Wang T, Huang Q, Zhou Y-R, Yu B-W, et al. Benzbromarone aggravates hepatic steatosis in obese individuals. Biochim Biophys Acta Mol Basis Dis. 2018;1864(6):2067-77.

53. Fornasier G, Taborelli M, Francescon S, Polesel J, Aliberti M, De Paoli $P$, et al. Targeted therapies and adverse drug reactions in oncology: the role of clinical pharmacist in pharmacovigilance. Int J Clin Pharm. 2018;21.

54. Zhang W, Doherty M, Bardin T, Pascual E, Barskova V, Conaghan P, et al. EULAR evidence based recommendations for gout. Part II: management. Report of a task force of the EULAR standing committee for international clinical studies including therapeutics (ESCISIT). Ann Rheum Dis. 2006;65(10):1312-24

55. Walter-Sack I, de Vries JX, von Bubnoff A, Pfleilschifter V, Raedsch R. Biotransformation and uric acid lowering effect of benzbromarone in patients with liver cirrhosis - evidence for active benzbromarone metabolites? Eur J Med Res. 1995;1(1):16-20.

\section{Publisher's Note}

Springer Nature remains neutral with regard to jurisdictional claims in published maps and institutional affiliations. 